\title{
Role of p.r.n. antimuscarinic medication in the treatment of antipsychotic-induced extrapyramidal movement disorders
}

\author{
Luke Birmingham, Norman McClelland and Caroline Bradley
}

The antimuscarinic drugs procyclidine, orphenadrine, benzhexol and benztropine are used in psychiatric practice to alleviate the extrapyramidal effects of antipsychotic medication. These drugs may be prescribed on a regular, asrequired or single-dose basis. They are usually taken by mouth, in tablet or liquid form, but for rapid onset of action procyclidine and benztropine can be given by intramuscular or intravenous injection.

\section{Role of antimuscarinic medication in the treatment of antipsychotic- induced extrapyramidal effects}

Antimuscarinic drugs are most effective for treating antipsychotic-induced Parkinsonism (rigidity, bradykinesia and tremor) and dystonic reactions, including oculogyric crisis. Akathisia is unlikely to be helped by antimuscarinic medication, and tardive dyskinesia can be exacerbated by these drugs (Blaisdell, 1994; Ebel, 1994; Barnes \& Phillips, 1996; Holloman \& Marder, 1997).

Antimuscarinic drugs should not be regarded as the mainstay for the treatment of antipsychoticinduced extrapyramidal movement disorders. The most reliable and effective treatment is to reduce the dose of the antipsychotic medication and, mental state permitting, maintain the patient on the lowest possible dose thereafter. An alternative is to substitute an atypical antipsychotic drug. Such drugs, which have fewer extrapyramidal side-effects, may be useful for patients in whom conventional antipsychotics continue to cause troublesome extrapyramidal side-effects despite a reduction in antipsychotic dosage coupled with the addition of antimuscarinic medication. If the above measures fail and it proves necessary to prescribe antimuscarinic drugs, these should be used sparingly. Whenever possible, antimuscarinic drugs should be prescribed on an 'as-required' (p.r.n.) as opposed to a regular basis.

\section{Prophylactic, regular and p.r.n.} prescribing of antimuscarinic drugs

The prophylactic use of antimuscarinic medication for patients on long-term antipsychotic medication is not recommended (World Health Organization, 1990). Antimuscarinic drugs have side-effects of their own and they exacerbate the antimuscarinic effects of antipsychotic drugs. When given regularly, in combination with antipsychotic medication, they can reduce the therapeutic activity of antipsychotic medication (Bamrah et al. 1986) and predispose to the development of tardive dyskinesia (Barnes \& Phillips, 1996). Furthermore, antimuscarinic medication can cause an acute toxic state characterised by agitation and psychotic symptoms (Johnson et al, 1981). Long-term use may be associated with memory impairment (Calev. 1984), and antimuscarinic drugs may be misused for their euphoriant properties (Pullen et al. 1984: Marken et al, 1996).

Regular antimuscarinic medication should not be given unless it is clear that without this the patient would continue to suffer from troublesome extrapyramidal symptoms. If the patient suffers only intermittently from extrapyramidal symptoms, 'as-required' antimuscarinics coupled with patient education may suffice. In circumstances where an antipsychotic depot preparation is being given on a regular basis. antimuscarinic medication may be required only for a few days after each dose of depot is given. Under these circumstances a limited p.r.n. supply can be dispensed. 


\section{Regulating the use of antimuscarinic medication}

When antimuscarinics are used, the prescription should be reviewed on a regular basis. In many cases, extrapyramidal side-effects associated with commencing or increasing the dose of antipsychotic medication are self-limiting and resolve within a matter of weeks (Barnes \& Phillips, 1996), therefore it is worthwhile attempting to reduce and discontinue antimuscarinics prescribed for newly emergent extrapyramidal disorders after a period of three months.

The instructions accompanying a p.r.n prescription require accurate interpretation, and a working knowledge of antimuscarinic and antipsychotic drug properties is required if antimuscarinics are to be used only when indicated. Patients can receive considerable amounts of muscarinic medication via the 'as-required' route. Therefore, p.r.n. prescriptions should be monitored as closely as prescriptions for antimuscarinics given on a regular basis. Educating patients about the side-effects of antipsychotic drugs and when to use 'as-required' doses of an antimuscarinic agent is also important. In addition, because nursing staff charged with the responsibility of dispensing p.r.n. doses of antimuscarinic drugs are not always familiar with the indications and contraindications for giving this medication (Birmingham et al, 1999), it is worthwhile ensuring that staff receive training on this subject and that guidelines are available for them to consult.

Education of doctors, psychiatric nursing staff and patients should help to reduce inappropriate prescribing and dispensing of p.r.n. antimuscarinic medication. However, it is also advisable for doctors and nurses to continually monitor their practice. The following guidelines can be used for this purpose:

(a) If the patient asks for p.r.n. antimuscarinic medication, clarify the reason for the request.

(b) If the patient wants p.r.n. medication for 'side-effects', clarify the nature of these side-effects. Some patients use the euphoriant properties of antimuscarinic drugs to help overcome the depressant activity of antipsychotic medication, and others may be misinformed.

(c) Make an objective assessment of the severity of the patient's antipsychoticrelated side-effects and ensure that antimuscarinic medication is given only when indicated, that is, for acute extrapyramidal effects.

(d) Finally, before prescribing/dispensing. consider whether anything else should be taken into account with the patient, such as tendency to misuse substances.
In the longer term, it is important to monitor the patient's use of p.r.n. medication. If excessive quantities are being taken, this should be looked into. If significant amounts are being taken for genuine reasons, then other treatment avenues should be explored.

Avoiding the use of antimuscarinic agents when they are not indicated and regulating their use when they are prescribed help to reduce the risk of patients developing unwanted, medicationrelated side-effects. This not only reduces unpleasant or stigmatising side-effects experienced by the patient but, with poor compliance being an issue for many patients taking antipsychotic drugs, steps taken to minimise unwanted medication-related effects may tip the balance in favour of compliance with treatment.

\section{References}

BAMRAH, J. S., KUMAR, V., KRSKA, J., et al (1986) Interactions between procyclidine and neuroleptic drugs. Some pharmacological and clinical aspects. British Journal of Psychiatry. 149. 726-733.

BARNes. T. R. E. \& PHILuPS, M. A. (1996) Antipsychoticinduced extrapyramidal symptoms. Role of anticholinergic drugs in treatment. CNS Drugs, 6. 315-330.

Birmingham, L.. MCClelland, N. \& BRADLey. C. (1999) p.r.n. prescribing and administration of antimuscarinic medication. Psychiatric Bulletin. 23, 184-185.

BLAISDELL, G. D. (1994) Akathisia. A comprehensive review and treatment summary. Pharmacopsychiatry. 27. 139-146.

CALEV. A. (1984) Anticholinergic drugs and memory. British Journal of Psychiatry, 143, 422-423.

EBEL. H. (1994) Therapy of neuroleptic-induced extrapyramidal movement disorders. Neurology. Psychiatry \& Brain Research, 2. 140-151.

HOLLOMAN, L. C. \& MARDER. S. R. (1997) Management of acute extrapyramidal effects induced by antipsychotic drugs. American Joumal of Health-System Pharmacy. 54. 2461-2477.

Johnson, A. L., Hollister, L. E. \& Berger, P. A. (1981) The anticholinergic intoxication syndrome: diagnosis and treatment. Journal of Clinical Psychiatry. 42, 313-317.

MARKEN, P. A., STONER, S. C. \& BUNKER, M. T. (1996) Anticholinergic drug abuse and misuse. CNS Drugs, $\boldsymbol{\delta}$. 190-199.

Pullen, G. P.. Best, N. R. \& Maguire, J. (1984) Anticholinergic drug abuse: a common problem? British Medical Journal. 289, 612-613.

WORLD HEalth ORganization HEads of CENTrEs Collaborating in WHO Co-ordinated Studies ON BIOLOGICAL ASPECTS OF MENTAL ILLNESS (1990) Prophylactic use of anticholinergics in patients on long-term neuroleptic treatment. A consensus statement. British Journal of Psychiatry. 166, 412.

*Luke Birmingham, Lecturer in Forensic Psychiatry; Norman McClelland, Nursing Lecturer in Forensic Psychiatry; and Caroline Bradley, Specialist Registrar in Forensic Psychiatry, Reaside Clinic, Birmingham Great Park, Bristol Road South, Birmingham B45 9BE

*Correspondence 